\title{
ELECTRICAL CHANGES IN DEEPER CORTICAL STRUCTURES DURING BALANCED GENERAL ANESTHESIA WITH THE AIM ON INHALATION ANESTHETICS EFFECTS
}

\author{
Fischer $\mathbf{M}^{1}$, Drobny $\mathbf{M}^{1}$, Saniova $\mathrm{B}$, Bakosova $\mathrm{E}^{1}$, Hamzik J ${ }^{2}$, Schnierer $\mathbf{M}^{3}$, \\ Osinova D. ${ }^{1}$
}

\author{
${ }^{1}$ Clinic of Anesthesiology and Intensive Medicine, Comenius University, Jessenius Faculty of Medicine and \\ University Hospital Martin, Slovakia \\ ${ }^{2}$ Clinic of Thoracic Surgery, Comenius University, Jessenius Faculty of Medicine and University Hospital Martin, \\ Slovakia \\ ${ }^{3}$ Clinic of Gastroenterological Internal Medicine, Comenius University, Jessenius Faculty of Medicine and \\ University Hospital Martin, Slovakia
}

\begin{abstract}
There are many theories that attempt to explain the mechanisms of the effects of inhalation anesthetics - from simpler, pursuing individual effects of anesthetics on the level of the ion channels, to more complex that are looking for uniform global changes in brain activity common to several agents. However, we still don't have satisfactory and adequate conclusions.

We examined a sample of 39 patients undergoing thoracic surgery at the Clinic of Thoracic Surgery under general anesthesia (GA) and we registered their electroencephalographic (EEG) signals before and during operation. After induction of GA by intravenous (i.v.), we used inhalation anesthetics to maintain GA. We used sevoflurane (SEV) in 20 patients and desflurane (DES) in 19 patients. Then we obtained the EEG data and processed them through mathematical and statistical analysis, to discover any changes of electrical activity in the brain during thoracic surgery under GA.

The era of digital recording EEG and present possibilities of modern computer techniques allow quantitative analysis of obtained data. We performed the analysis with the software LORETA (low resolution brain electromagnetic tomography). It is a relatively new research method, which in a similar way as computed tomography (CT) or magnetic resonance imaging (MRI) displays even deeper brain electrical activity, which is hiddeen for a classical EEG approach.

We described the general changes in brain electrical activity of the deeper cortical structures within the traditional frequency bands (d, q, a, b and g) during GA at $5 \mathrm{~mm}$ spatial resolution. We have shown that the source of the well-known cortical EEG changes after the effect of used inhalation anesthetics is caused by changes situated in the deeper brain structures, particularly the limbic system. Significant changes occurred in the cingulate gyrus for most of an EEG frequency ranges. When comparing the data of patients anesthetised with SEV and DES we found similar changes within the $d$ and q rhythms and then the global changes of EEG activity followed during GA.
\end{abstract}

Key words: general anesthesia, EEG, inhalation anesthetics, LORETA, deeper cortical structures

\section{INTRODUCTION}

The satisfying mechanism of action how volatile anesthetics work is still unknown (1). The same question exists about exact localization of these mechanisms in the brain. We still don't possess sufficient option how to estimate the depth of GA for clinical purposes. Usually the anesthesiologists rely only on clinical signs of patient (heart rate, blood pressure, movement of body parts, size of pupils or skin changes) what is a cheap but inaccurate method. Even measuring the fraction of used inhalation anesthetic in exhaled gas mixture is insufficient. Even there has been a recent development of few devices that relies on

Address for correspondence:

Fischer M., Clinic of Anesthesiology and Intensive Medicine, Comenius University, Jessenius Faculty of Medicine and University Hospital Martin, Kollárova 2, 036 59, Martin, Slovakia, Phone:+421 434203 683; e-mail: mudr.fischer.martin@gmail.com 
intraoperative analysis of EEG signal to estimate the depth of anesthesia but they still lack required accuracy (2). Therefore, a lot of criticizing voices exist among researchers and professionals (3). Besides that new digitalized computational analysis the EEG signal brought us the possibility to quantitative asses the changes of electrical activity in the brain, it correlates them with the activity of a particular amounts of used inhalation anesthetics in exact locations. The main goal of this work is to monitor the basic changes in electrical activity of the whole cortical brain regions that are induced by use of inhalation anesthetics during GA. There are other intentions that tend to compare effects of the common similar inhalation anesthetics - in our case this counts for sevoflurane and desflurane. Subsequent analyses should clarify the way of action and its location.

\section{METHODS}

We examined 39 patients who underwent elective different thoracotomic surgery at the Clinic of Thoracic Surgery. The group consisted of 21 men and 18 women with an average age of 62 years. All patients were operated under general anesthesia (GA). We excluded patients with previous head injury concomitant with loss of consciousness, addicted to drugs or alcohol, known intolerance or no standard response to an anesthetic, patients with neurological or psychiatric illness or who are currently abusing psychotropic medication.. Institutional review board approval and written informed consent were obtained in all cases.

The GA was performed by a specialist anesthetist at usual manner in accordance with the clinic practice of balanced anesthesia. EEG recording did not interfere with the common practice of general anesthesia.

For the standard procedure of anesthesia, the anesthesiologist used intravenous agents for the fast introduction of a patient into the GA with preceding 2 minutes of preoxygenation with 100\% oxygen. Propofol was used as the main intravenous anesthetic for induction of GA, followed by opioid sufentanil and nondepolarising neuromuscular muscle relaxants (rocuronium, atracurionium or cis-atracurium). Further, after the intubation, followed the administration of inhalation anesthetics to the patient. We used sevoflurane in 21 patients (SEV) and desflurane for 18 patients (DES). The next table presents demographical information of the observed group (Table 1). In 28 cases the anesthetist supplemented the GA with intravenous bolus of ketamine short time before first surgical incision.

Table 1: Demographic information of observed subgroups of patients according to used inhalation anesthetic.

Numbers represent the average values of patients. SEV $=$ sevoflurane, DES $=$ desflurane, MAC $=$ average minimal alveolar concentration of used inhalation anesthetic.

\begin{tabular}{|l|l|l|l|l|l|}
\hline & number & age & males & females & MAC \\
\hline SEV & 20 & 61.3 & 8 & 12 & 0.76 \\
\hline DES & 19 & 62.6 & 11 & 8 & 0.83 \\
\hline
\end{tabular}

Then was the depth of GA maintained on the demanded surgical level by further administration of inhalation anesthetics after considering the clinical condition of the patient - the blood pressure, heart rate and ventilation.

The EEG record was continually done during whole GA and the doses of all used i.v. medications, current phases of operation or anesthesia, and all other factors which might affect our recorded data were noted down. The amounts of used inhalation anesthetics were meas- 
ured by the anesthetic machine in form of minimal alveolar concentration (MAC), which was calculated from the analysis of expired gasses in 5 minutes lasting intervals.

EEG signal was recorded just after the patients entered the operating theater prior to their morning premedication. We obtained short sample of artefact free EEG data while patients were holding closed eyes. This was used as a reference value of EEG signal without any influence of anesthetics. Then the recording continued until the end of anesthesia just before the patients were extubated.

For EEG recording we used standard EEG caps with 19 electrodes distributed according to the ninternational 10-20 system. The EEG cap was chosen according to circumference of patients head. After applying some conductive gel, we kept the impedance of electrodes on such level when it did not exceed $25 \mathrm{~kW}$. The recording contained raw data ranging from $0.1 \mathrm{~Hz}$ to $500 \mathrm{~Hz}$.

All data used in this study were derived from the referential EEG montage, where the EEG recording software used a virtual electrode as a reference that was computed out of an average derived from all used hardware electrodes. By using this setup we eliminated the appearance of possible total artifacts, which could influence whole record (4).

Further processing the EEG data followed later. Statistical analysis and graphical illustration of the results were obtained by software eLORETA (exact LORETA). This software is capable to reconstruct and display not only superfitial cortical, but also deeper cortical electric active areas of grey matter from provided standard EEG data. This is achieved by means of linear and quantitative power spectral analysis (5). By this approach eLORETA is capable to compute functional model of brain electrical activity in 3 spatial dimensions (6). The computations could be compared to computed tomography (CT) or magnetic resonance imaging (MRI). Lower number of sensory input points defined by number of used EEG electrodes (7) and mutual electromagnetic interference combined with shielding surrounding anatomic structures of the head cause the obtained spatial resolution of results is much lower when compared to CT or MRI techniques. Deeper structures have tendency to occur blurred. On the other hand, the very good temporal resolution is measured in order of milliseconds. This makes the eLORETA method ideal for following the rapidly changing dynamics of brain functions (8). All analyzed EEG data were obtained by continual monitoring during standard clinical practice. We visually checked the obtained EEG signals and selected 10 representing segments, each lasting 2 seconds. These segments were artifacts free and we repeated this for every phase of operation and for every patient.

According to the defined purposes of this study we designed the following specific phases of a thoracic surgical intervention:

control phase - state before operation representing basic resting state of the brain activity; patient lying with closed eyes in quiet environment without premedication or any other medications with possible chance to affect consciousness.

phase of deep general anesthesia - state somewhere in the middle of the operation representing deep surgical anesthesia; i.v. medications used for induction into GA has already negligible effect on current EEG recording (100 minutes after the use of i.v. anesthetic agents during the induction to GA, SD 10 minutes) and the patient was in such a stage of anesthesia, when adequate and steady input of inhalation anesthetics matched the objective surgical requirements.

In our cases the use of opioids during the maintanance of balanced GA did not have effect on the EEG recording. Only high doses were asociated with the rise of slow d activity. When considering continuous supplementation of the analgetic sufentanile within clinical dosage (in general $0.5 \mu \mathrm{g} / 30$ minutes), its effects on EEG are negligible (9).

We needed to choose comparable parts of anesthesia. The depth of current GA could be approximately estimated by measuring the MAC. MAC is the concentration of used inhalation anesthetics in alveolar space that causes the voluntary movement reaction for standard nociception stimuli (e.g. surgical skin incision) disappears in 50\% of cases. Every inhalation anesthetics has its proprietary MAC value estimated experimentally that illustrates its potency. 
Direct measurement of MAC in clinical environment is complicated, so anesthetic machine is able to estimate it after calculations based on concentration of inhalation anesthetics in exhaled gasses. It is measured in volume percentage of inhaled gas mixture and for sevoflurane it was experimentally estimated on $2.05 \%$ and for desflurane on $6 \%$ (according to manual and technical specifications of used anesthetic machine). Numeric MAC values showed in real time on the screen of anesthetic machine and MAC values used in this work represents the ratio of the measured exhaled volume \% of used inhalation anesthetic to experimentally estimated MAC value for every substance.

We analyzed the EEG activity in next frequency ranges (4):

delta $(\delta)=1-3.5 \mathrm{~Hz}$

theta $(\theta)=4-7.5 \mathrm{~Hz}$

alfa $(\alpha)=8-13 \mathrm{~Hz}$

beta $(\beta)=14-29 \mathrm{~Hz}$

gamma $(\gamma)=30-50 \mathrm{~Hz}$

First step in data analysis was the normalization. We used so-called "subject-wise" normalization, which is a part of the eLORETA software package. With this step we normalized the measured power for all time segments on the same level, what removed interindividual difference amongst the patients that could contaminate our data (e.g. different conductivity of the skin, thickness of the bones in the skull and so on).

Then we divided the EEG data into groups according to phase of operation and created graphical outputs in form of a topographical 3D maps of brain for the different frequency ranges and in different cross sections. These pictures represented computed 3D distribution of sources of electric currents - the standardized current density (J) measured in $\mu \mathrm{A} / \mathrm{mm}^{2}$, which were calculated by proprietary eLORETA algorithms. With these pictures we were able to determine the size and localization of the maximal $J$ and its distribution to surroundings.

To be able to display changes of the electrical activity during the GA we needed to compute the difference in between the resting state before operation and the deep surgical anesthesia. We used the statistical module included in eLORETA sowtware to perform a paired voxel-by-voxel independent sample F-ratio tests (similar to log of ratio of averages), based on eLORETA log-transformed current density power. This is how we made independent multiple comparisons in between 2 groups: for every patient, and for every voxel. This makes huge amount of tests $=39$ patients * 6239 voxels of grey tissue in brain, what makes 243 321 t-tests for whole group within every frequency range. This is how we estimated the critical threshold that every voxel needs to exceed by its $\mathrm{F}$ value to prove its significancy. Because we used multiple t-tests we needed to correct the calculated critical thresholds of F-values and related probabilistic p-values. We did this in form of statistical non-parametric testing, so we computed 5000 randomizations for every evaluated voxel.

Definitive topographic maps showed us the significant difference between compared groups. The result was the representation of location and size of maximal and minimal differences. Visual outputs are pictures depicting 3D topographical maps of the electrical brain activity changes in a form of computed F-values as a result of statistical testing all voxels in brain. With this approach we are able to display maximal positive and maximal negative changes in between compared states.

To allow a free comparison of the patients within the whole file we needed to find out whether the used inhalation anesthetics were administered in the same doses. After securing the same dosage we should attain the similar depth of anesthesia and the followed EEG data should to be comparable. We evaluated the amount of used inhalation anesthetics by comparing MAC values of patients anesthetized by sevoflurane and MAC values in the group anesthetized by desflurane.

MAC values were statistically processed and evaluated in the statistical software MYSTAT. We used Lilliefors test to find that the data did not have normal distribution. Subsequent Wilcoxon test did not find a significance amongst SEV and DES groups for basic probability $p=0.03$ therefore we are presenting them together. Average MAC of used inhalation anesthetic (SEV + DES) was 0.8 with standard deviation 0.24. 


\section{RESULTS}

Next set of pictures represents the statistically significant difference between the control phase and the phase of general anesthesia. It is the result of comparison all 39 patients anesthetized by sevoflurane and desflurane.

Huge changes during anesthesia in brain functioning corresponds to the discovery of multitude significant changes (written description of Fig. 1). It covers the changes in electrical activity of brain during GA for individual frequency ranges when compared to control state before operation.
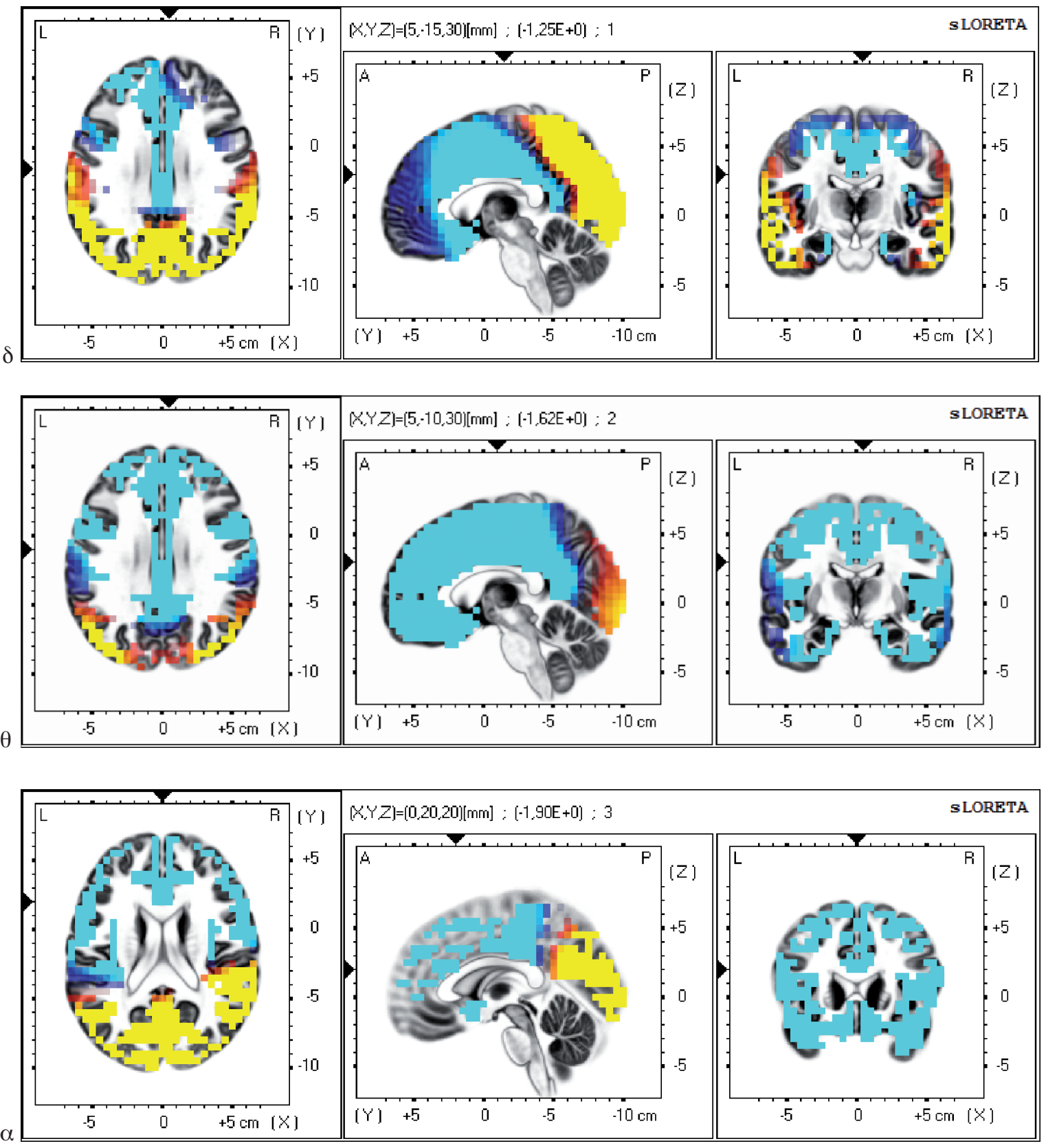

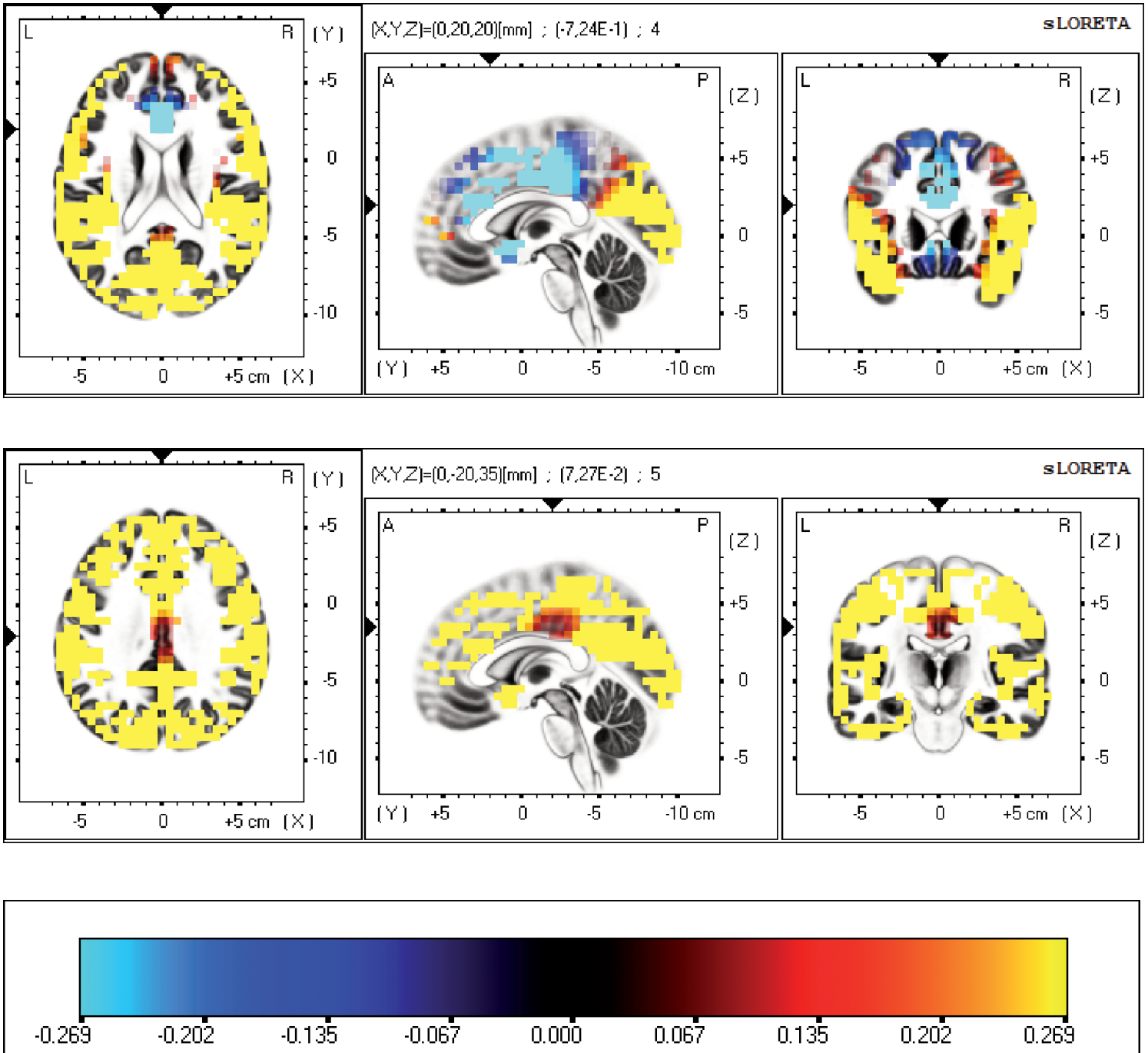

Fig. 1: Positive differences in the brain electrical activity during GA. The figure shows results of statistical testing. Every voxel has its color according to the statistically computed F-value within every frequency range. The view is optimized to show maximal significant difference during the GA when compared to the resting state before the operation. Every frequency rhythm has its own set of 3 topographic 3D maps representing by slices in standard anatomical planes (from left to right): horizontal, sagittal and coronal plane. Spatial orientation is noted in the upper corners of every map $(\mathrm{L}=$ left, $\mathrm{R}=$ right, $\mathrm{A}=$ anterior, $\mathrm{P}=$ posterior $)$. On the lower and right borders of every map there is a scale (in centimeters) defining the grid of the 3D space. On the upper and left borders there are black triangle- shaped arrows pointing to the spot with the maximal negative activity. This represents the places that are more active during the GA. On the bottom there is a color scale, which assigns the hue according to the statistical significance. In this situation, every significant voxel needs to overcome the threshold of 0.269 (F-value), which was computed for probability of error of the first order $\mathrm{p}<0.01$. Therefore every cyan colored voxel has statistical significant difference. The yellow voxels represent the significantly more active spots during the resting phase. 
Delta rhythm was before anesthesia significantly more active in occipital, parietal and temporal lobe with its maximum set in the superior parietal lobule with slightly lateralization to the left. Delta activity increased during the anesthesia in the depth of limbic lobe slightly exceeding to the surface frontally with right lateralization. Maximum of this activity was found in limbic lobe - cingulate gyrus.

Theta rhythm was before operation significantly more powerful at the occipito-temporal surface with maximum in the posterior part of middle temporal gyrus. After the onset of anesthesia we saw massive significant movement of theta activity to the limbic area, spreading to the area of whole frontal lobe and through the surface of the insula to the deeper parieto-occipital areas, with small right predominance. The maximum of this activity was situated in cingulate gyrus.

Alpha rhythm showed significantly higher activity before operation across whole range of grey brain matter of the occipital lobe, with temporo-parietal propagation while lightly affecting even deep posterior structures of the limbic system. Maximal activity was localized into medial occipital gyrus with right lateralization. During the GA alpha rhythm was significantly stronger in the depth of limbic lobe while it influenced whole frontal lobe, insula and then it partially expanded to the temporo-parietal area. Maximum of alpha activity was in the anterior cingulate cortex, slightly more expressed to the left.

Beta rhythm significantly dominated before operation across almostwhole range of brain grey matter with predominance of superficial structures. Maximal activity emerged from the medial temporal gyrus bilaterally. Then the beta activity spread to all directions, predominantly frontally and to depth (from temporo-occipital area, through deeper structures of the insula and limbic system) to the frontal lobe. The brain showed during anesthesia significantly higher activity only in relatively small area of the limbic lobe with light irradiation to the deeper structures of the frontal lobe (even to the surface on small area of the medial frontal gyrus). Maximum was placed in the depth of anterior cingulate cortex.

Maximal activity of gamma rhythm before the GA was situated in the medial temporal gyrus with slight left predominance. From there it spread significantly more or less evenly to all directions to all brain structures, even to the depth. The spreading took place through insular area and partially through limbic system to the parietal lobe and from there to whole occipital and frontal lobes. There was only small area of brain which did not show significantly higher activity, placed deep in the cingulate gyrus. Not any area of brain showed statistically significant higher activity during anesthesia, however the last remains of gamma activity could be found in the posterior cingulate cortex.

We determined the amount of spatial scope of these changes by counting the number of voxels that showed significant differences during the GA. The biggest scope was found in the gamma range (the global decrease of electrical activity except the small area deep in the limbic lobe - cingulate gyrus). Then follows the alpha range (massive anteriorization of alpha activity -movement from occipital area to the whole frontal region), beta range (global occipito-temporo-parieto-frontal activity preoperatively moved to the medial areas of the brain hemispheres in the limbic system), theta (massive movement from the superficial occipitoparietal areas preoperatively to the frontal, orbito-frontal and neighboring areas activated from the inner parts of limbic system). The least spatial large movements of electrical activity were found in the delta range (from the occipito-parieto-temporal areas preoperatively to mostly deeper limbic and frontal areas).

\section{DISCUSSION}

We can clearly see the spectral changes in electric activity, the anteriorization of that activity and overall slowing down of the rhythms even by simple visual estimation of the eLORETA results during GA (10). 
When comparing phase before to during anesthesia, we can detect anteriorization of electrical activity. But we can also detect some sort of similar changes in $\theta$ and $\delta$ rhythms. Building on these statements we can say that observable changes in electrical activity of the brain are occuring in accordance with already published papers $(11,14)$. Except this, we can observe changes caused by inhalation anesthetics in much higher resolution and even in the deeper cortical structures. These findings could shed some light to explanation of such changes.

Generally, we could say that during the GA were activated predominately deeper parts of the brain in the limbic lobe. Mostly by the slower rhythms $-\alpha$ and $\theta$ range that activate the surroundings from depth to the surface. This activity comes from the cingulate gyrus for $\theta$ and $\delta$ rhythms or more accurate from the posterior part of the cingulate gyrus for $\alpha$ and $\beta$ rhythms. The biggest growth of electrical activity in this area happened for a rhythm, followed by $\theta$ rhythm, which affected other fronto-parietal areas by the greatest spatial extent. Then comes $\delta$ range with much lower promotion to the surrounding areas and finally $\beta$ rhythm, which radiates only minimally to the surroundings and promotes to the brain cortex in a limited space.

$\gamma$ rhythm did not manifest higher activity during GA in any area, but it persisted with moderate power in the cingulate gyrus posterior part probable as a fundamental underlying oscilation for subconscious cognition connected with „Aha - reflex“ (12). The persisted $\gamma$-band in the posterior segment of cingulate gyrus is in good correlation with experimental discovery of Stancak and Fallon (13) that long lasting chronic pain is capable to cause the atrophy of the cingulate gyrus posterior segment. Therefore cognitive function carried-out by $\gamma$-activity is possible surrogate of delayed cognition assured also in deep GA unconsciousness stages that is able also under such condition to percept chronic sensory bombardment by nociceptor signals provoking apoptosis of posterior segment cingulate gyrus neurocyte or even the whole neuropil.

\section{CONCLUSIONS}

We managed to describe basic changes in electrical activity of the brain when using inhalation anesthetics with such spatial resolution, which was not published in the literature before. Our results link cortical electrical changes with the massive changes that are emerging from the deep brain structures. The source of these actions is localized into the limbic system, specifically to the cingulate gyrus (the different parts are dedicated for the different frequency ranges). Our findings are partially in line with the uniform theory of action of the inhalation anesthetics that are opening the problem not only from the global macrostructural field of view, but also from the probable intracellular microtubular protein conformation oscilation as a primary source of gamma rhythm underlying animal lucid conscious awareness. However, these results are only partial.

Lately, there has been a shift in the EEG evaluation data from the classical qualitative methods towards the newer quantitative approach (pEEG-processed EEG). It was allowed by the recent increase of the cheap computing power recently. eLORETA, as one of the latest complex software methods, appears to be a promising way to process EEG in many interdisciplinary fields. We still lack the unambiguous uniform theory explaining how the general anesthetics, and especially inhalation anesthetics acts (10). However, in the case of potent inhalation anesthetics (including SEV and DES) we can expect quite similar general effects on brain functioning, even when considering the different mechanisms of actions among them on the more detailed level of ion channels. Even when the various studies are discovering different pharmacodynamic principles of inhalation anesthetics on a scale of ion channels, we can expect more frequent addition of eLORETA method and similar approaches on these findings in the near future, particularly in more scientifically controlled clinical trials. 
The specific literature in this case of potent inhalation anesthetics (including SEV and DES) probably expects quite similar mechanisms of action in more general scope, because for the general uniform thinking of the most anaesthesiologists. They does not take quite in account the basic quantum physical principles working also in the human brain as a material substrate, for example superposition, quantum entanglement in spite of the brain $\mathrm{s}$ moisture and temperature creating a noise. Even when the various studies are discovering different pharmacodynamic principles on a smaller scale, like ion channels, we can expect more frequent addition of eLORETA method in such discoveries in the near future.

\section{REFERENCES}

1. Campagna JA, Miller WK, Forman SA. Mechanisms of Actions of Inhaled Anesthetics. New England Journal of Medicine [online]. 2003; 348(21):2110-2124.

2. Jagadeesan N, Wolfson M, Chen Y, Willingham M, Avidan MS. Brain monitoring during general anesthesia. Trends in Anaesthesia and Critical Care [online]. 2013, 3(1):13-18.

3. Jordan D, Stockmanns G, Kochs EF, Pilge S, Schneider G. Electroencephalographic order pattern analysis for the separation of consciousness and unconsciousness: an analysis of approximate entropy, permutation entropy, recurrence rate, and phase coupling of order recurrence plots. Anesthesiology [online]. 2008, 109(6):1014-1022.

4. Donald L, Schomer, da SILVA FL. Niedermeyer's Electroencephalography: Basic Principles, Clinical Applications, and Related Fields. Sixth edition. Philadelphia: LWW. ISBN 978-0-78178942-4.

5. Pascual-Margqui RD. Discrete, 3D distributed, linear imaging methods of electric neuronal activity. Part 1: exact, zero error localization. arXiv:0710.3341 [math-ph, physics:physics, q-bio] [online]. 2007.

6. Fuchs M, Kastner J, Wagner M, Hawes S, Ebersole JS. A standardized boundary element method volume conductor model. Clinical Neurophysiology: Official Journal of the International Federation of Clinical Neurophysiology. 113(5):702-712.

7. Jurcak V, Tsuzuki D, Dan I. 10/20, 10/10, and 10/5 systems revisited: Their validity as relative head-surface-based positioning systems. NeuroImage [online]. 2007, 34(4):1600-1611.

8. Pascual-Marqui RD. Standardized low-resolution brain electromagnetic tomography (sLORETA): Technical details. Methods and Findings in Experimental and Clinical Pharmacology. 2002, 24(Suppl D): 5-12. .

9. Butterworth J, Mackey DC, Wasnick J. Morgan and Mikhail's Clinical Anesthesiology, 5th edition. 5 edition. New York: McGraw-Hill Medical. ISBN 978-0-07-162703-0.

10. Miller R D, Eriksson LI., Fleisher L, Wiener-Kronish JP, Young WL. Miller's Anesthesia 2 volume set: Expert Consult - Online and Print, 7e. 7 edition. Philadelphia, PA: Churchill Livingstone. ISBN 978-0-443-06959-8.

11. John ER, Prichep LS, Kox W, Valdés-Sosa P, Bosch-Bayard J, Aubert E, Tom M, DiMichele F, Gugino LD. Invariant Reversible QEEG Effects of Anesthetics. Consciousness and Cognition [online]. 2001, 10(2):165-183.

12. Kandel ER, Dudai Y, Mayford MR. The molecular and systems biology of memory. Cell [online]. 2014, 157(1):163-186.

13. Stancak A, Fallon NB. Emotional modulation of experimental pain: a source imaging study of laser evoked potentials. Frontiers in Human Neuroscience [online]. 2013, 7:552.

14. Brown EN, Lydic R, Schiff ND. General Anesthesia, Sleep, and Coma. New England Journal of Medicine [online]. 2010, roč. 363, č. 27, s. 2638-2650. ISSN 0028-4793. Dostupné na: doi:10.1056/NEJMra0808281

Received: July,10,2015

Accepted: December,5,2015 